\title{
Self-detection of MIR QCL frequency combs (Withdrawal Notice)
}

Pierre Jouy, Gustavo Villares, Johanna Wolf, Filippos Kapsalidis, Mattias Beck, et al.

Pierre Jouy, Gustavo F. Villares, Johanna Wolf, Filippos Kapsalidis, Mattias Beck, Jérôme Faist, "Self-detection of MIR QCL frequency combs (Withdrawal Notice)," Proc. SPIE 10123, Novel In-Plane Semiconductor Lasers XVI, 101230Y (21 April 2017); doi: 10.1117/12.2254786

SPIE. Event: SPIE OPTO, 2017, San Francisco, California, United States 


\section{Self-detection of MIR QCL frequency combs (Withdrawal Notice)}

Pierre Jouy, Gustavo F. Villares, Johanna Wolf, Filippos Kapsalidis, Mattias Beck, Jérôme Faist ETH Zürich (Switzerland)

Proceedings of SPIE Vol. 10123, 101230Y (2017)

Online Publication Date: 20 April 2017

Withdrawn from Publication: 12 June 2017

Conference Date: 28 January 2017

Conference Location: San Francisco, California, United States

Conference Title: Novel In-Plane Semiconductor Lasers XVI

Conference Chairs: Alexey A. Belyanin, Peter M. Smowton

This paper, originally published on 20 April 2017, has been withdrawn per author request. 